\title{
RADICAL APPROACH IN BCH-ALGEBRAS
}

\author{
EUN HWAN ROH
}

Received 2 October 2002 and in revised form 4 November 2004

\begin{abstract}
We define the notion of radical in $\mathrm{BCH}$-algebra and investigate the structure of $[X ; k]$, a viewpoint of radical in $\mathrm{BCH}$-algebras.
\end{abstract}

2000 Mathematics Subject Classification: 06F35, 03G25.

1. Introduction. In 1966, Imai and Iséki [8] and Iséki [9] introduced two classes of abstract algebras: BCK-algebras and BCI-algebras. It is known that the class of BCKalgebras is a proper subclass of the class of BCI-algebras. In 1983, $\mathrm{Hu}$ and $\mathrm{Li}[5,6]$ introduced a wide class of abstract algebras: $\mathrm{BCH}$-algebras. They have shown that the class of BCI-algebras is a proper subclass of the class of $\mathrm{BCH}$-algebras. They have studied some properties of these algebras.

As we know, the primary aim of the theory of $\mathrm{BCH}$-algebras is to determine the structure of all BCH-algebras. The main task of a structure theorem is to find a complete system of invariants describing the $\mathrm{BCH}$-algebra up to isomorphism, or to establish some connection with other mathematics branches. In addition, the ideal theory plays an important role in studying BCI-algebras, and some interesting results have been obtained by several authors [1, 2, 3, 4, 11, 14, 15]. In 1992, Huang [7] introduced nil ideals in BCI-algebras. In 1999, Roh and Jun [13] introduced nil ideals in BCH-algebras. They introduced the concept of nil subsets by using nilpotent elements, and investigated some related properties.

In this note, we define the notion of radical in $\mathrm{BCH}$-algebra, and some fundamental results concerning this notion are proved.

2. Preliminaries. A BCH-algebra is a nonempty set $X$ with a constant 0 and a binary operation “ $*$ " satisfying the following axioms:

(1) $x * x=0$,

(2) $x * y=0$ and $y * x=0$ imply $x=y$,

(3) $(x * y) * z=(x * z) * y$

for all $x, y, z \in X$. A BCH-algebra $X$ satisfying the identity $((x * y) *(x * z)) *(z * y)=$ 0 and $0 * x=0$ for all $x, y, z \in X$ is called a BCK-algebra. We define the relation $\leq$ by $x \leq y$ if and only if $x * y=0$.

In any BCH-algebra $X$, the following hold: for all $x, y \in X$,

(4) $(x *(x * y)) \leq y$,

(5) $x \leq 0$ implies $x=0$,

(6) $0 *(x * y)=(0 * x) *(0 * y)$, 
(7) $x * 0=x$,

(8) $0 *(0 *(0 * x))=0 * x$.

A nonempty subset $S$ of BCH-algebra $X$ is called a subalgebra of $X$ if $x * y \in S$ whenever $x, y \in S$.

A nonempty subset $I$ of BCH-algebra $X$ is called an ideal of $X$ if $0 \in I$ and if $x * y, y \in I$ imply that $x \in I$. It is possible that an ideal of a $\mathrm{BCH}$-algebra may not be a subalgebra.

3. Main results. In what follows, the letter $X$ denotes a $\mathrm{BCH}$-algebra unless otherwise specified.

DEFINITION 3.1. For any $x \in X$ and any positive integer $n$, the $n$th power $x^{n}$ of $x$ is defined by

$$
x^{1}=x, \quad x^{n}=x *\left(0 * x^{n-1}\right) .
$$

Clearly $0^{n}=0$.

THEOREM 3.2. For any $x \in X$ and any positive integer $n$,

$$
(0 * x)^{n}=0 * x^{n}
$$

Proof. We argue by induction on the positive integer $n$. For $n=1$ there is nothing to prove. Assume that the theorem is true for some positive integer $n$. Then using (6) we have

$$
\begin{aligned}
(0 * x)^{n+1} & =(0 * x) *\left(0 *(0 * x)^{n}\right) \\
& =(0 * x) *\left(0 *\left(0 * x^{n}\right)\right) \\
& =0 *\left(x *\left(0 * x^{n}\right)\right)=0 * x^{n+1} .
\end{aligned}
$$

Definition 3.3. [10] In a BCH-algebra $X$, the set $A^{+}:=\{x \in X \mid 0 \leq x\}$ is called a positive part of $X$ and the set $A(X):=\{x \in X \mid 0 *(0 * x)=x\}$ is called an atom part of $X$. Further an element of $A(X)$ will be called an atom of $X$.

In the following theorem we give some properties of BCK-algebras.

THEOREM 3.4. If $X$ is a BCH-algebra, then the positive part $A^{+}$of $X$ is a subset of the set $\left\{x \in X \mid x^{2}=x\right\}$.

Proof. Let $x \in A^{+}$. Then we have $x^{2}=x *(0 * x)=x * 0=x$, and hence $A^{+} \subseteq\{x \in$ $\left.X \mid x^{2}=x\right\}$.

COROLlary 3.5. If $X$ is a BCK-algebra, then $X=\left\{x \in X \mid x^{2}=x\right\}$.

In [10], Kim and Roh proved $A(X)=\{0 *(0 * x) \mid x \in X\}=\{0 * x \mid x \in X\}$.

Note that $A(X)$ is a subalgebra of $X$ and $((x * y) *(x * z)) *(z * y)=0$ for all $x, y, z \in A(X)$, and hence $A(X)$ is a $p$-semisimple BCI-algebra. Thus by [12] we have the following property: for any $a, b \in A(X)$ and any positive integer $n$, we have $(a * b)^{n}=$ $a^{n} * b^{n}$.

Hence the following corollary is an immediate consequence of Theorem 3.2. 
TABLE 3.1

\begin{tabular}{c|cccc}
\hline$*$ & 0 & $a$ & $b$ & $c$ \\
\hline 0 & 0 & $c$ & 0 & $a$ \\
$a$ & $a$ & 0 & $a$ & $c$ \\
$b$ & $b$ & $c$ & 0 & $a$ \\
$c$ & $c$ & $a$ & $c$ & 0 \\
\hline
\end{tabular}

COROLLARY 3.6. For any $x$ in a BCH-algebra $X$ and any positive integer $n$,

(i) $0 * x^{n} \in A(X)$,

(ii) $0 *(x * y)^{n}=\left(0 * x^{n}\right) *\left(0 * y^{n}\right)$.

DEFINITION 3.7. Let $R$ be a nonempty subset of a BCH-algebra $X$ and $k$ a positive integer. Then define

$$
[R ; k]:=\left\{x \in R \mid x^{k}=0\right\},
$$

which is called the radical of $R$.

We know that, in general, the radical of an ideal in $X$ may not be an ideal.

EXAMPLE 3.8. Let $X=\{0, a, b, c\}$ be a BCH-algebra in which $*$-operation is defined as in Table 3.1. Taking an ideal $R=X$, then $[R ; 3]=\{0, a, c\}$ is not an ideal of $X$ since $b * a=c \in[R ; 3]$ and $b \notin[R ; 3]$.

THEOREM 3.9. Let $S$ be a subalgebra of a BCH-algebra $X$ and $k$ a positive integer. If $x \in[S ; k]$, then $0 * x \in[S ; k]$.

Proof. Let $x \in[S ; k]$. Then $x^{k}=0$ and $x \in S$. Thus by Theorem 3.2 we have

$$
(0 * x)^{k}=0 * x^{k}=0, \quad 0 * x \in S,
$$

and hence $0 * x \in[S ; k]$.

This leaves open question, if $R$ is a subalgebra of $X$ and $0 * x \in[R ; k]$, then is $x$ in $[R ; k]$ ? The answer is negative. In Example 3.8, $[X ; 3]$ is a subalgebra of $X$ and $0 * b \in$ $[X ; 3]$, but $b \notin[X ; 3]$.

Definition 3.10 [10]. For $e \in A(X)$, the set $\{x \in X \mid e * x=0\}$ is called the branch of $X$ determined by $e$ and is denoted by $A(e)$.

THEOREM 3.11. Let $k$ be a positive integer and $A(X)=X$. Then

$$
A(e) \cap[X ; k] \neq \varnothing \Longrightarrow A(e) \subseteq[X ; k] .
$$

Proof. Suppose that $A(e) \cap[X ; k] \neq \varnothing$, then there exists $x \in A(e) \cap[X ; k]$. Thus by Theorem 3.9, we have

$$
e=0 *(0 * x) \in[X ; k] .
$$

Let $y \in A(e)$, then $y \in[X ; k]$ since $0 *(0 * y)=e \in[X ; k]$, and hence $A(e) \subseteq[X ; k]$. 
THEOREM 3.12. For any positive integer $k$ and $A(X)=X$,

$$
[X ; k]=\underset{x \in[X ; k]}{\bigcup} A(0 *(0 * x))=\underset{e \in A(X) \cap[X ; k]}{\cup} A(e)=\underset{e \in[A(X) ; k]}{\cup} A(e) .
$$

Proof. $A(X) \cap[X ; k]=[A(X) ; k]$ is obvious. By Theorem 3.11, we have $x \in A(0 *$ $(0 * x)) \subseteq[X ; k]$ for all $x \in[X ; k]$, and so there exists $e=0 *(0 * x) \in A(X) \cap[X ; k]$ such that $x \in A(e) \subseteq[X ; k]$. Therefore we obtain

$$
[X ; k]=\underset{x \in[X ; k]}{\bigcup} A(0 *(0 * x))=\underset{e \in A(X) \cap[X ; k]}{\cup} A(e)=\underset{e \in[A(X) ; k]}{\cup} A(e) .
$$

ACKNOWLEDGMENT. The author is deeply grateful to the referees for the valuable suggestions and comments.

\section{REFERENCES}

[1] B. Ahmad, On classification of BCH-algebras, Math. Japon. 35 (1990), no. 5, 801-804.

[2] M. A. Chaudhry, On BCH-algebras, Math. Japon. 36 (1991), no. 4, 665-676.

[3] M. A. Chaudhry and H. Fakhar-Ud-Din, Ideals and filters in BCH-algebras, Math. Japon. 44 (1996), no. 1, 101-111.

[4] W. A. Dudek and J. Thomys, On decompositions of BCH-algebras, Math. Japon. 35 (1990), no. $6,1131-1138$.

[5] Q. P. Hu and X. Li, On BCH-algebras, Math. Sem. Notes Kobe Univ. 11 (1983), no. 2, part 2, 313-320.

[6] _ On proper BCH-algebras, Math. Japon. 30 (1985), no. 4, 659-661.

[7] W. P. Huang, Nil-radical in BCI-algebras, Math. Japon. 37 (1992), no. 2, 363-366.

[8] Y. Imai and K. Iséki, On axiom systems of propositional calculi. XIV, Proc. Japan Acad. 42 (1966), 19-22.

[9] K. Iséki, An algebra related with a propositional calculus, Proc. Japan Acad. 42 (1966), 26-29.

[10] K. H. Kim and E. H. Roh, The role of $A^{+}$and $A(X)$ in BCH-algebras, Math. Japon. 52 (2000), no. 2, 317-321.

[11] S. Y. Kim, Q. Zhang, and E. H. Roh, On nil subsets in BCH-algebras, Math. Japon. 52 (2000), no. 2, 285-288.

[12] J. Meng and S. M. Wei, Periods of elements in BCI-algebras, Math. Japon. 38 (1993), no. 3, 427-431.

[13] E. H. Roh and Y. B. Jun, On properties of nil subsets in BCH-algebras, submitted to Indian J. Pure Appl. Math.

[14] E. H. Roh, Y. B. Jun, and Q. Zhang, Special subset in BCH-algebras, Far East J. Math. Sci. (FJMS) 3 (2001), no. 5, 723-729.

[15] E. H. Roh, S. Y. Kim, and Y. B. Jun, On a problem in BCH-algebras, Math. Japon. 52 (2000), no. 2, 279-283.

Eun Hwan Roh: Department of Mathematics Education, Chinju National University of Education, Jinju 660-756, Korea

E-mail address: ehroh@cue.ac.kr 


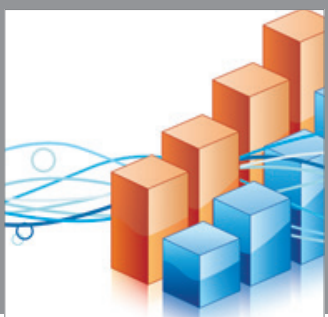

Advances in

Operations Research

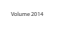

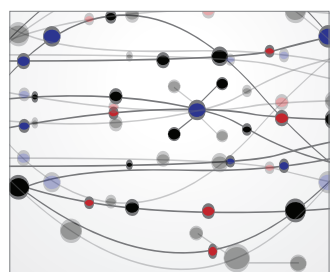

\section{The Scientific} World Journal
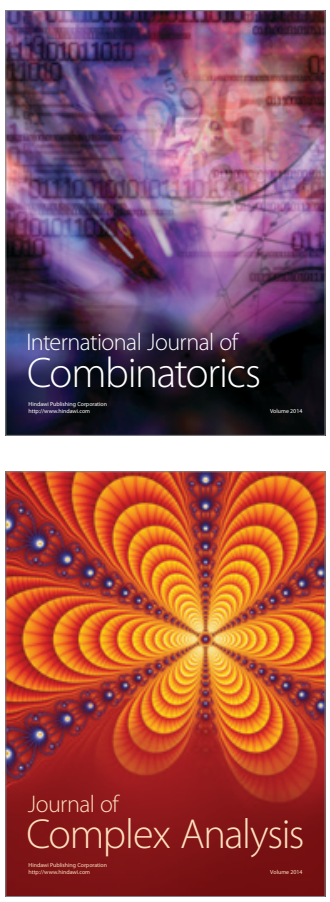

International Journal of

Mathematics and

Mathematical

Sciences
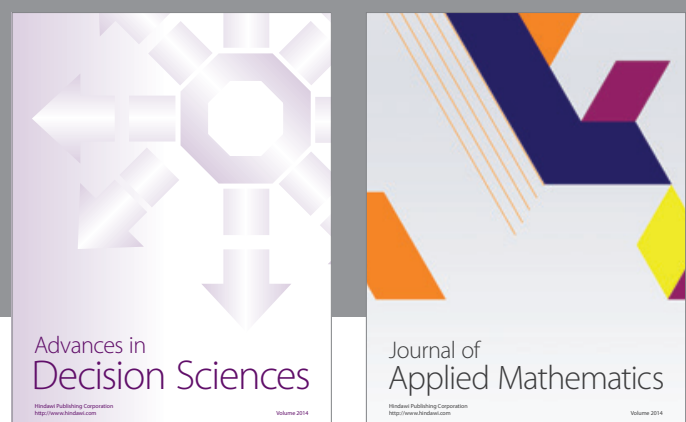

Journal of

Applied Mathematics
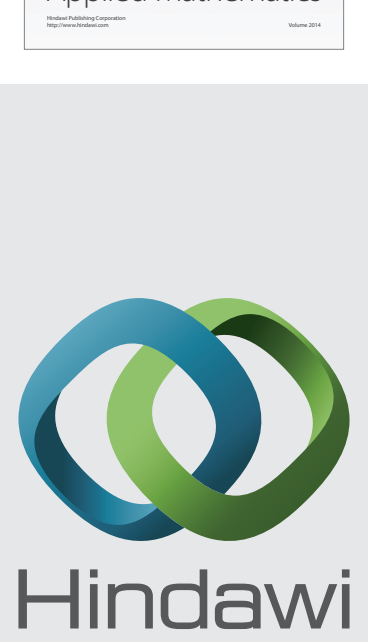

Submit your manuscripts at http://www.hindawi.com
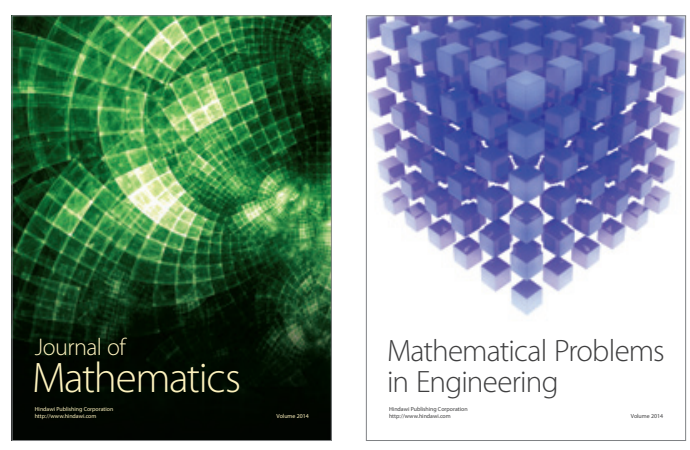

Mathematical Problems in Engineering
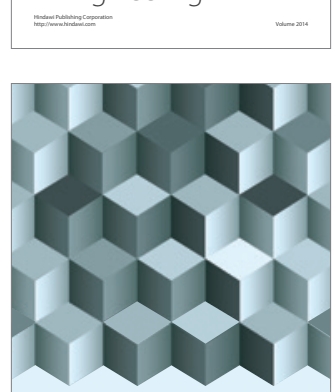

Journal of

Function Spaces
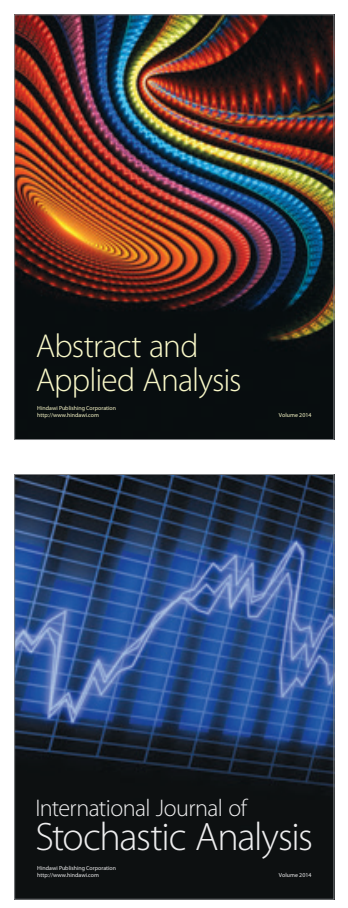

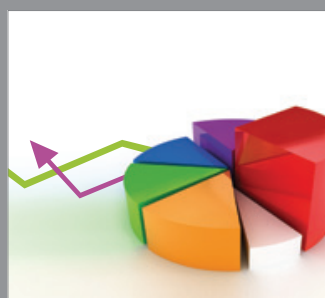

ournal of

Probability and Statistics

Promensencen
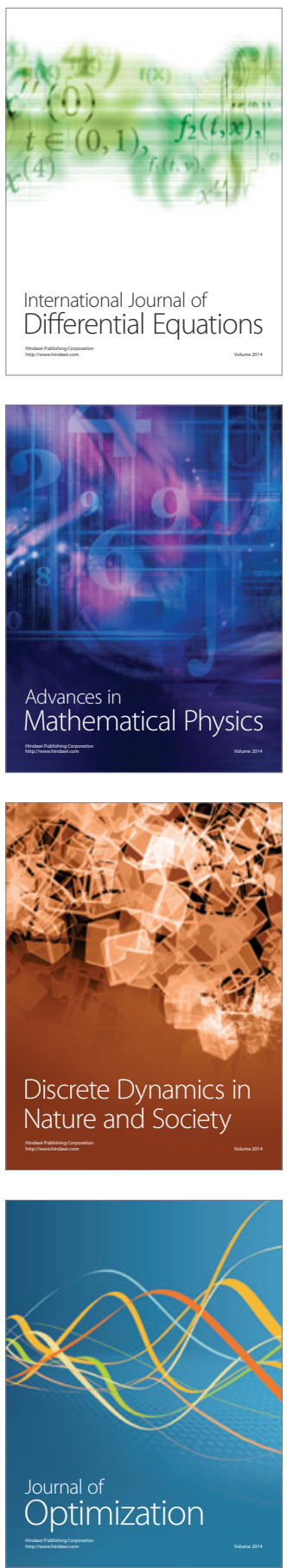